\title{
HUMAN FACTORS RESEARCH ON PERFORMANCE-BASED NAVIGATION INSTRUMENT PROCEDURES FOR NEXTGEN
}

\author{
Divya C. Chandra and Rebecca J. Grayhem \\ United States Department of Transportation \\ Volpe National Transportation Systems Center, Cambridge, MA
}

\begin{abstract}
Area navigation (RNAV) and required navigation performance (RNP) are key components of performance-based navigation (PBN). Instrument procedures that use RNAV and RNP can have more flexible and precise paths than conventional routes that are defined using ground-based navigation aids. As a result, RNAV and RNP routes can enhance operational safety, efficiency, and access.
\end{abstract}

At the United States Department of Transportation Volpe Center, we are identifying and documenting human factors issues associated with implementing $\mathrm{PBN}$ instrument procedures for the Federal Aviation Administration (FAA). This effort supports the FAA's transition to the Next Generation Air Transportation System (NextGen) by providing recommendations to promote easier and more reliable use of PBN instrument procedures. In this paper, we explain why human factors research is needed and provide examples of research issues. For example, new procedures may result in increased visual complexity of charts, which in turn could increase pilot workload and the potential for error.

We are also conducting analyses and experiments to understand and improve the usability of aeronautical charts for PBN instrument procedures. Our work in this area is summarized in this paper. More information can be found at www.volpe.dot.gov/coi/hfrsa/ahf/ip/library.html. The website has links to our latest research products. It also supports other human factors research teams by listing and summarizing publicly available reports on related topics.

\section{Introduction}

The United States (US) Federal Aviation Administration (FAA) and the International Civil Aviation Organization (ICAO) are transitioning to performance-based navigation (PBN) operations. Area navigation (RNAV) procedures are fundamental to this effort. RNAV allows an aircraft to fly directly between points in space without relying on conventional ground-based navigation aids (e.g., by using satellite-based navigation). Required Navigation Performance (RNP) is a refinement of RNAV that includes on-board monitoring and alerting to ensure that the actual performance of the navigation system keeps the aircraft position within established criteria. RNP allows more precise path design, which is particularly useful for developing approach procedures to runways. For example, RNP approaches often include radius-to-fix (RF) path segments (i.e., precisely curved legs) to avoid obstacles. Further information on RNAV and RNP can be found in a variety of FAA handbooks and reports $[1-5]$.

Instrument procedures based on RNAV, including those with RNP segments, offer safety enhancements along with new levels of flexibility to negotiate terrain, airspace, and environmental considerations. More RNAV procedures, with and without RNP segments, are being developed each year in order to support PBN $[5,6]$. The FAA is committed to developing RNAV and RNP procedures, particularly those with large operational benefits, as part of its response to recommendations made by RTCA [7] and in response to legislative requirements.

There are human factors concerns associated with the design, depiction, and implementation of RNAV and RNAV (RNP) procedures because they can result in paths that are complex to fly and depict. These routes typically require the assistance of a Flight Management System (FMS) to negotiate precise speed, altitude, and lateral path constraints. Therefore, the FAA's broad human factors research requirement is to ensure that performance-based

U.S. Government work not protected by U.S. copyright 
instrument procedures are usable by appropriately qualified pilots.

Volpe Center researchers are helping the FAA program managers to identify, scope, plan, and coordinate research projects that address the wide variety of human factors impacts that could arise with performance-based operations. In addition, the Volpe Center is leading research to address one key issue, the usability of charts for RNAV and RNAV (RNP) procedures.

In the next section, we provide additional background on RNAV and RNAV (RNP) procedure design considerations and training requirements. Then we describe our progress on the research planning and coordination tasks. Finally, we summarize progress on our chart usability research and present planned work.

\section{RNAV and RNP}

Figure 1, from the FAA website, illustrates the design efficiencies that RNAV and RNP afford relative to conventional routes. RNAV alone allows for additional airspace efficiency, as seen by the rectangular airspace boundaries in place of the trapezoidal boundaries required for conventional navigation aids. Paths can be more precise with RNAV, as illustrated by the reduced width of the rectangles. With RF legs, curved paths can be created to avoid terrain or other areas such as special use airspace or noise abatement regions. RNP paths can be even more precise than RNAV paths because of the aircraft monitoring and alerting capabilities, as illustrated by the even narrower rectangles. Because RNP is essentially a type of RNAV procedure, we refer to RNAV routes with RNP segments as RNAV (RNP) procedures.

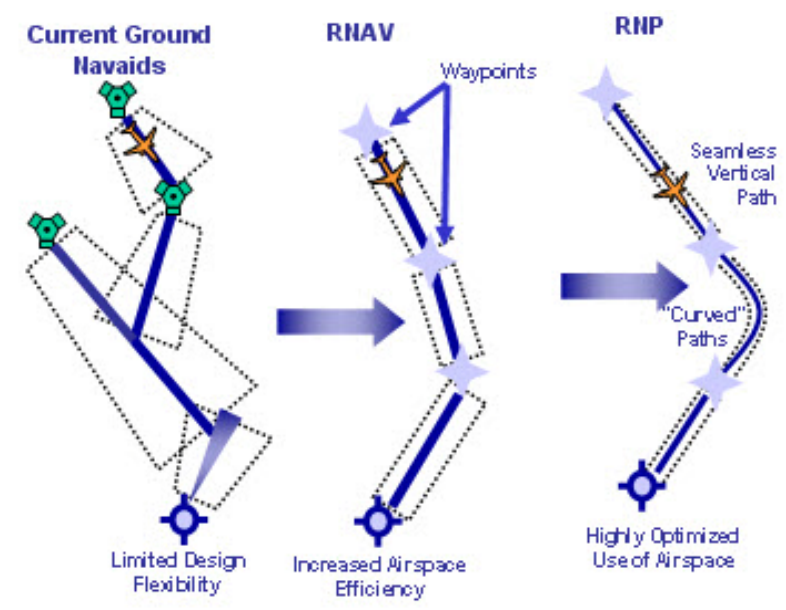

Figure 1. Conventional, RNAV, and RNP Routes

Training requirements for RNAV procedures are contained in [8], for RNP operations and barometric vertical navigation in [9], and for RNAV (RNP) Authorization Required (AR) procedures in [10]. Pilots must be familiar with both text and graphical depictions of RNAV and RNAV (RNP) procedures. From these, they must be able to understand the flight path, determine equipage requirements for the individual procedure, and be able to use and understand RNAV and RNP terminology, as well as specific air traffic phraseology. Pilots must also be able to understand and use RNAV and RNP information that is system-specific, such as flight deck automation and alerting interfaces. Pilots must be able to operate RNAV equipment appropriately (e.g., initialize system position and use the FMS to monitor the flight path and adhere to speed and/or altitude constraints associated with the procedure). Finally, pilots must be able to execute contingency procedures in case of RNAV and RNP failures.

\section{Research Planning and Coordination}

In order to understand the research needs in this area, Volpe Center compiled and reviewed relevant published and unpublished materials. We also consulted with industry and government subject matter experiments. The products of this effort are a literature library and a 5-year research plan that defines areas for research. 


\section{Literature Review}

There is relatively little published literature on the specific topic of human factors issues related to PBN. One early document is a technical report in which the authors compile, review, and categorize a long list of issues [11]. The issues list was gathered from a review of voluntarily submitted aviation safety reports, attendance at professional meetings and conversations with subject matter experts. Some of the issues are coordination or process issues, while others are research issues that could be addressed through data collection. One of the key recommendations from this report is that research is needed to develop comprehensive and specific human factors guidelines for the design of RNAV and RNAV (RNP) instrument procedures that take into account flight crew and controller performance. They acknowledge that this goal can only be achieved through a series of research studies, not one study alone.

In a related report [12], the authors analyzed reports pertaining to RNAV procedures from the Aviation Safety Reporting System (ASRS) database, a public online database of voluntary reports that is managed by the National Aeronautics and Space Administration (NASA). They identified 124 reports filed between 2000 and mid-2005 related to RNAV Standard Instrument Departure (SID) and Standard Terminal Arrival Route (STAR) procedures at seven specific airports. They broadly categorized key issues as being related to air traffic operations, pilot interpretation of procedures, and procedure design challenges with aircraft automation and charting.

An updated review of ASRS reports related to RNAV and RNAV (RNP) was published in 2010 [13]. This report reviews 285 relevant voluntary reports filed between January 2004 and April 2009 at 17 airports. The bulk of these reports (202) pertained to SID procedures and 69 pertained to STAR procedures. Only14 reports mentioned approach procedures. This may be because there were relatively few operators who flew RNAV (RNP) approach procedures during the period studied. Some of the issues identified in these reports concerned the operation of the FMS and Air Traffic Control (ATC) phraseology. Chart and procedure issues were also found in the set, but in fewer reports.
We identified many sources of information that were outside of the traditional literature in our search. Some of the published documents contain technical reference material about the design and use of instrument procedures (e.g., FAA and ICAO documents); these are not focused on human factors issues, but they do provide important background and context. A technical report produced annually presents the prevalence of different procedures and aircraft equipage trends [6]. A list of publicly available reports on these topics (with summaries) is posted at the Volpe Center public website (www.volpe.dot.gov/coi/hfrsa/ahf/ip/library.html).

This library was developed to support other human factors research teams who are beginning work in this area. Additional background and current information about RNAV and (RNP) can be gathered at various open meetings sponsored by industry and the government.

\section{Research Plan}

Volpe Center prepared a 5-year research plan describing human factors research and development needs and objectives regarding charting and instrument procedures for the FAA by reviewing documents from our literature review and by consulting with subject matter experts. The plan contains a list of topics divided into sections based on whether the projects are active, proposed, or closed. Background information is provided to explain the topic further and to justify why the topic is important. Previous research is cited where possible. An initial plan was developed in FY11 and an update was released in FY12. Annual updates are planned. The research plan will help to ensure that funded research projects align with the FAA's NextGen implementation goals. The latest plan is available at our public website.

The scope of the research plan is broad. This is necessary because charts are used by all types of operators and because PBN must be accessible to all operators as well. For example, the research plan encompasses RNAV and RNAV (RNP) operations as well as hybrid procedures that combine RNAV and conventional navigation requirements (e.g., an RNAV (RNP) missed approach procedure for an otherwise conventional approach procedure). Research must address all types of flight operations under Title 14 of the Code of Federal Regulations 
(14 CFR): private operations including piston-engine and jet operations (Part 91), air taxi and charter operations (Part 135), commercial scheduled service (Part 121), single pilot, and military operations. Each of these operations presents their own set of challenges.

The research must also consider the interactions between flight deck design, implementation, flight operations, and ATC. These interrelationships were identified in the analyses of ASRS reports [12], [13] and they will be important to understand as RNAV and RNAV (RNP) procedures are used more often.

One of the core areas in the research plan is the usability of charts, both electronic and paper. Work in the areas of chart content, chart format, and overall chart usability will lay the foundation for further research. A long-term goal of the charting research is to develop human factors guidance for instrument procedure designers, which would be the most efficient point in the process to address the complexity of chart depictions.

For electronic charts specifically, there are additional research areas, such as de-cluttering of moving map displays and integration of chart and procedure information onto moving map displays. We also need to understand the impacts of transitioning continuously between different charts and scales (e.g., from departure to en route operations) to provide appropriate information for each phase of flight.

There are three technology drivers for this research on aeronautical charts. First, of course, is PBN, which brings challenges for human performance. In particular, $\mathrm{PBN}$ routes are more precise laterally, and in many cases, there are more vertical (altitude and speed) constraints as well. There can also be many notes and supplementary information to review and process with PBN routes. Some of these notes are routine, but others are not, and it can be hard to determine at a glance which ones are the most critical.

A second technology driver, independent of PBN, is the shift from paper to electronic chart media. Electronic charts come in a variety of forms. Some are just scanned copies of paper charts that are "pre-composed" (i.e., not interactive, other than in that they can be zoomed and panned). However, some electronic charts are database driven [14] [17]. These charts are highly customizable and can be created and modified in real-time. One advantage of data-driven charts is that they can be customized to correspond to the planned route of flight in the FMS. For example, elements that are unrelated to the route of flight could be suppressed to reduce display clutter. Database driven charts will support a number of significant changes, such as the integration of different chart types (en route, arrival, and departure) into a single electronic product that changes the data shown based on the location of the aircraft.

The third technological shift is that more chart information is beginning to be integrated onto installed flight deck displays such as the moving map display. Although the moving map display is not designed to replace a paper chart, it can show some of the data relevant to the flight procedure. For example, the map display may show the altitude constraints at various points along an approach procedure that is entered into the FMS. This shift will bring other human factors issues to the forefront, such as prioritization of information on a shared display (i.e., one that supports additional applications). As more chart and procedural data are integrated in to the flight deck, the placement of that information, potentially across more than one display, will also need to be explored so that information is easily accessible and integrated when necessary.

Open issues regarding chart content for both electronic and paper media include, for example, the use of and need for the profile view of an approach procedure. With the procedure programmed into the FMS, do pilots refer to the profile view for their final approach headings and altitudes? Also, because there is an increasing amount of vertical information shown on arrival and departure procedures, would it be beneficial (or even feasible) to present a profile view on these procedures. The depiction of altitude information, including vertical angles is especially confusing in current charts, and human factors research could provide guidance on how to make this information clearer to pilots. Procedure naming has also been a complicated and increasingly relevant issue that might benefit from human factors research and data. Current procedure naming conventions do not adequately communicate the performance and functional requirements of $\mathrm{PBN}$ procedures. An 
updated international naming convention is under discussion.

Other areas of research in the 5-year plan are more long term. For example, one topic is the charting of dynamic clearances, which are routes that may change based on weather or traffic conditions. Optimal Profile Descents (OPD's), for example, can be thought of as a dynamic clearance. The exact routing for the OPD could change day to day, but some elements of the procedure are likely to remain consistent. Would it be useful to provide any published guidance for these types of clearances, or would the entire clearance be provided to crews every time it is issued?

Air-ground integration issues for RNAV operations are another area for future research. For example, controller phraseology has already been an area of concern for descend via and climb via clearances. Also, charts are used not only by flight crews, but also by air traffic controllers. Controllers use charts for training and may allocate responsibilities to different staff based on how procedures are designed. Communication between flight crews and controllers about their equipage to fly PBN routes may also be an issue. Current equipment suffixes do not convey the full range of possible configurations.

Finally, charting of and the use of en route airways based on RNAV (Q routes for high-altitude operations above $18,000 \mathrm{ft}$ and $\mathrm{T}$ routes for lowaltitude operations below $18,000 \mathrm{ft}$ ) may require some human factors evaluations. These routes are now depicted on aeronautical charts but to date no data have been collected to determine whether there is any confusion with conventional routes or whether users are able to identify and use $\mathrm{Q}$ and $\mathrm{T}$ routes without difficulty.

These research projects require well-coordinated teams whose members come from a variety of organizations and have a range of skills and perspectives. The teams could include members who are government employees, researchers, and private industry subject matter experts. Expertise is required in areas such as human factors, experiment design, and aviation. The aviation experience should include operational experience with both conventional and performance-based navigation, as well as a solid understanding of the underlying technologies.

\section{Chart Usability Research}

Our goal is to improve the usability of aeronautical charts for PBN, both in paper and electronic format. To date, we have considered the use of arrival, departure, and approach procedure charts by airline and corporate pilots.

We first studied existing charts to understand how they were constructed from a human factors point of view and to identify specific features that could potentially make the chart more difficult to use [18]. We also wanted to understand how the FAA develops instrument procedures so that we could recognize how and when it would be most effective to provide human factors guidance to instrument procedure designers.

Next, we designed and implemented a study to explore a new chart format option, in which a chart that depicted multiple paths is simplified by splitting the paths across multiple pages [19]. This study explored the question of whether separating procedure information across chart pages yielded a performance benefit or not. However, drawbacks of this modification were not explored.

These efforts are described in more detail below.

\section{Analyses}

In the first stage of our research on chart usability, we examined current charts. This work is documented in [18], which contains detailed information about the constraints that chart manufacturers face. Perhaps surprisingly, there are no FAA regulations regarding the content and design of charts that apply across all chart producers (private and government). However, standards have evolved within and across chart producers through cooperation and convention.

We first looked at current challenges and options for chart manufacturers. For this analysis, we assumed that the procedure design is fixed. Next, we reviewed several charts in order to discover what chart or procedure features may be contributing to operational issues. Finally, we explored the process for designing and implementing new instrument procedures. 
One of the charting challenges for RNAV and RNAV (RNP) procedures is that there is a lot of variation between procedures at different locations. Terrain, special use airspace, and other factors affect the overall shape of the path. Because RNAV and RNAV (RNP) allow more path design flexibility, there is inevitably more variation in how the route looks as well. One consequence of the flexibility offered by RNP is that it may take more time and care to read and review those charts to understand the procedure fully. Figures 2 and 3 illustrate this point.

Figure 2 shows the plan view of a simple conventional ground-based approach procedure. The image was extracted from the FAA chart for the Instrument Landing System (ILS) approach to Boise, Idaho Runway 10R. Notice the straight in approach path, represented by the arrowhead towards the runway. There are different ways to join the final approach, as indicated by the thin lines from Emett, Salla, and Renol. This chart is familiar to instrumentrated pilots and therefore easy to read; it looks like any other standard ILS procedure chart.

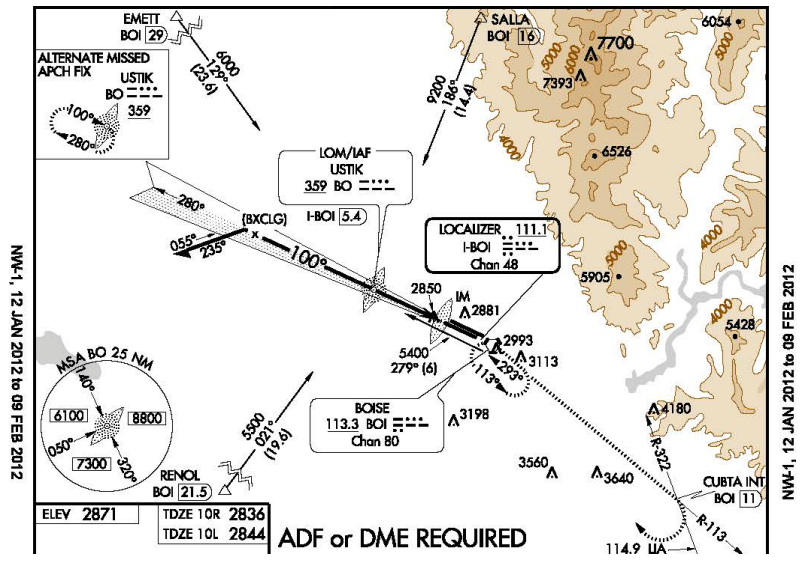

Figure 2. Plan View of an ILS Approach at Boise

Figure 3 shows a similar view of a corresponding RNAV (RNP) AR approach, which requires special aircraft and aircrew authorization. This image was extracted from the FAA chart for the RNAV (RNP) Z approach to Boise, Idaho Runway 10R. Notice the multiple approach paths, some of which include curved (RF) segments. There are also more path segments, more named points, and other information for each path. The scale of the plan view was adjusted to show a larger area, as seen by comparing the shaded areas of terrain in Figure 3 with those shown in Figure 2. Flying this procedure manually using only the chart as a source of path information would be extremely difficult, if allowed. In fact, typically, the aircraft must have at least the following equipment to fly an AR approach: dual Global Navigation Satellite System (GNSS) sensors, dual FMS, dual air data systems, dual autopilots, and a single Inertial Reference Unit (IRU) [10]. A moving map display is usually available as well. In this case, crews rely upon flight deck automation to fly the procedure correctly. The crew's job is to monitor the flight deck systems to ensure that it is flying the correct route and to manage unforeseen circumstances, such as an engine out during the approach.

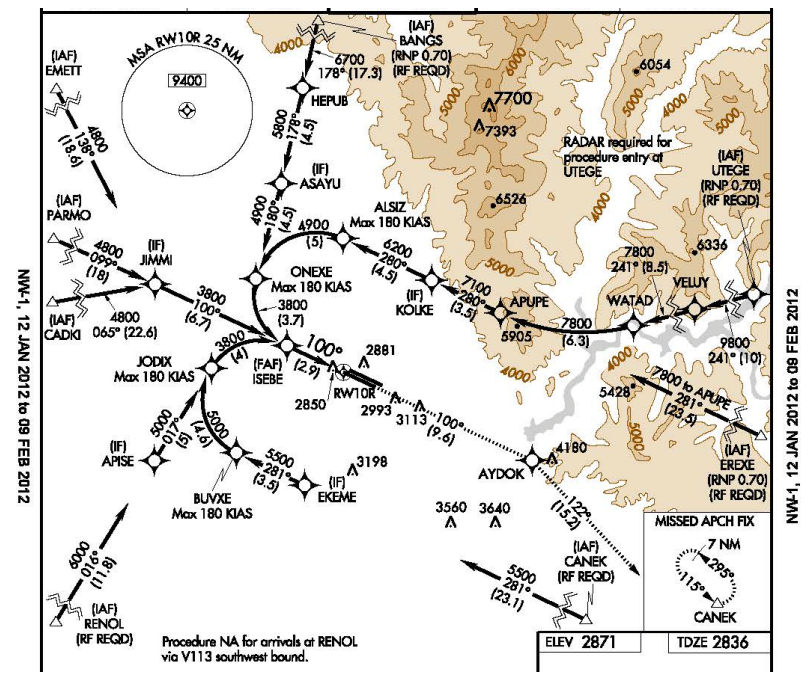

Figure 3. Plan View of an RNAV (RNP) Approach at Boise

Chart manufacturers use different techniques to optimize the depiction of detailed instrument procedures, whether or not they are based on RNAV and RNAV (RNP). Some of these techniques are the use of different fonts, color and shading, use of different scales, and different methods for standardizing the chart layout. For some of the most difficult charts, manufacturers have departed from their own standard practices for text, graphics, and even paper size. Figure 4, for example, shows the FAA chart for an RNAV (RNP) approach into Scottsdale, Arizona. In order to show the full approach path, not-to-scale insets are shown in the corners of the plan view. 


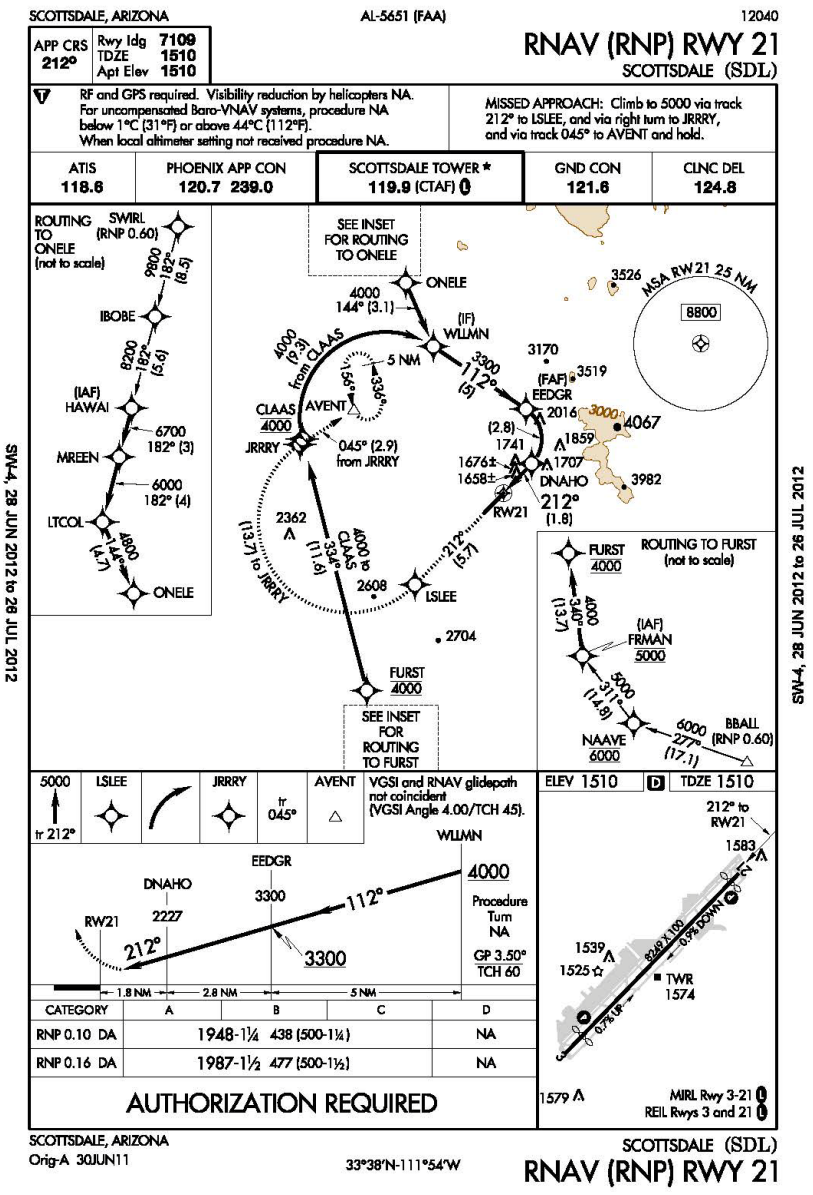

\section{Figure 4. RNAV (RNP) Approach in to Scottsdale, Arizona}

In another example, Jeppesen uses two larger than normal paper charts to show the Boise RNAV (RNP) approach procedure for Runway 28L. Both pages are foldouts, roughly twice the size of a standard chart. One page shows the full procedure and has a box around an area that is shown on the second page at a more detailed scale.

These types of deviations from normal conventions can create additional workload for pilots who are confronted with unique layouts. Unfamiliar formats could also increase the potential to misread information or miss information entirely.

After reviewing charting challenges and mitigation strategies, we were interested to know whether any objectively identifiable parameters of a procedure were correlated with difficulty of use. For this analysis, we compared two sets of RNAV and RNAV (RNP) charts in terms of different objective variables. One set of procedures was selected from those with operational issues noted in the ASRS review [13] or were highlighted by subject matter experts as being unusually complex; this was labeled the "Problematic" set. The second set of charts, labeled "Baseline," consisted of RNAV and RNAV (RNP) procedures from the Operational Evolution Partnership (OEP) airports (a set of 35 commercial airports in the US with significant activity) that did not appear in the Problematic set.

For approach charts, we recorded variables such as the number of flight paths shown, the total number of segments per path, and the number of curved segments. For SID and STAR procedures we recorded a slightly different set of variables (e.g., number of paths, number and types of altitude constraints, the types of altitudes depicted, distances along the different route segments, and overall distance for each path). For approaches, the main differences between the Problematic and Baseline sets were that the Problematic set had (a) more flight paths (b) more path segments, and (c) more curved (RF) segments. For STAR procedures, the Problematic set had more path segments and more altitude constraints. For SID procedures, the Problematic set had more flight paths. More information about this analysis is in [18] and [20] contains presents the full analysis.

Finally, we examined the process for creating instrument procedures. As mentioned earlier, process and coordination issues related to instrument procedures are also prevalent [11]. The process is complex, both in terms of the technical requirements and specifications, and in terms of the coordination required inside and outside the government. A key point is that instrument approach procedures are regulatory in nature and therefore have little flexibility in their design and use. Obstacle Departure Procedures (ODPs) are similar to approaches in that they are regulatory in nature because of the obstacle clearance requirements. In contrast, STAR and SID procedures are not regulatory and can be modified by ATC in day-to-day operations as needed. These differences are important for understanding the operational use of the various charts. Also, instrument approach procedures and ODPs are developed by FAA AeroNav Products, whereas arrival and departure procedures are developed by the Air Traffic Organization part of the FAA. These 
organizations use different software platforms to develop the different types of procedures, so they are truly created in different ways and meet very different needs.

\section{Chart Format Experiment}

This experiment was designed to measure whether separating paths across pages can result in benefits in terms of speed and accuracy of retrieving information. We hypothesized that it would be faster to retrieve information from the modified charts that show fewer paths. We recorded information retrieval times for both current and modified charts (i.e., charts modified to show fewer paths). We asked subjects to perform the task as quickly and as accurately as possible.

In order to increase the sensitivity of the experiment to finding performance benefits, only highly visually cluttered RNAV (RNP) approach and RNAV SID procedures were selected for the study. RNAV STAR procedures were not tested because they were less cluttered in general. Results of this study are reported in [19]. They will also be documented in a more complete government technical report in preparation that will include images of the modified charts that were developed for the study.

There was a large and statistically significant improvement for finding information from the modified charts in the study. For approach charts, pilots saved just over 6 seconds on average with the modified (simpler) charts. They saved 3 seconds on average with the modified SID charts. However, additional work is needed to establish whether the benefits remain, and to what extent, with less visually cluttered charts. For example, we expect that some charts will be simple enough that there is no performance benefit to creating multiple pages. That is, not all procedures will benefit from the modifications. For practical implementation, criteria by which procedures would be selected for this modification must be identified and defined so that the technique is not overused.

This experiment did not explore the practical disadvantages of separating paths across pages. Some drawbacks include having to search for the correct chart page within a set of separated pages, having more paper to carry in the flight deck (or more charts to choose from in a database), and the need for revisions to chart naming conventions. Pilots may also be less aware of the nearby paths that are not depicted but may be available for use. Additional drawbacks and limitations of the study are presented in [19].

\section{Charting Recommendations}

One of the planned products of this research program will be charting recommendations. To date, key recommendations for electronic charts are in a document on human factors considerations for Electronic Flight Bags (EFBs) from 2003 [21]. The electronic chart recommendations include topics such as zooming and panning, scale information, orientation, updates and backups. Recommendations regarding information priorities for data-driven charts are contained in [14].

There are also proposed recommendations from an industry committee under the PBN Aviation Rulemaking Committee (PARC) Chart Saturation Action Team, which were submitted to the PARC leadership in March 2010. The action team was formed in response to concerns about approach procedures such as those at Boise, Idaho, which present a lot of information in a constrained space. Some general recommendations from this working group were that:

- Additional human factors research is required.

- Charting implications should be considered during procedure design.

- Procedures should be able to be depicted uncluttered on a standard size US government chart, though the manufacture could still choose to use a larger chart.

More specific recommendations from the PARC Chart Saturation Action Team focused on design of approach procedures, not on chart depictions. For example, one recommendation is that design of STAR procedures should be considered when a new approach procedure is being developed. By considering both the STAR and approach at the same time, there can be more informed decisions about how and where to join the STAR to the approach procedure. Another recommendation concerns the 
use of multiple intermediate fix (IF) segments on approaches. Multiple IFs are the reason that the Boise approaches show multiple paths into the runway. The action team recommends that approach procedures with multiple IFs should be restricted to AR procedures; in other words, handling the complexity of multiple IF approaches should require special training.

\section{Planned Work}

Two tasks related to chart usability are planned for the next year. First, we will begin work in the area of electronic charts by assessing existing commercially available electronic charting software. Some of these applications run on mobile platforms (such as tablet computers) while others run on installed avionics. Our goal is to understand what features this type of software typically supports and what functions may need to be standardized or evaluated further.

Our second goal for the next year is to design another data collection effort to address two questions in more detail. First, how should charts be designed for compatibility with flight deck systems? Second, how are the various charting and procedure design options affected by the type of pilot and operations (e.g., corporate versus air transport)?

The study is still in the design stage; it will be a complement to the chart format study described above and in [19]. Our focus will again be on the content of the chart, not the media. However, here we would like to observe what information pilots actually use from the charts in a more realistic task and setting. In particular, the goal of this study is to explore how pilots use information from conventional and RNAV aeronautical charts in the context of a modern flight deck. Anecdotal evidence suggests that pilots are more dependent on flight deck systems now than they were in the past. Also, by assessing what information is used from the arrival and departure charts, we hope to develop insights on how these charts can be better standardized, in the same way that assessment of information use on approach charts in the 1990's, such as [22], led to improvements in the format of those charts.

\section{Summary}

In this paper we describe human factors research needs related to the implementation of PBN instrument procedures. We identified and documented these needs in a 5-year research plan for FAA human factors program managers. We also described progress on addressing the usability of charts for PBN procedures such as RNAV and RNAV (RNP) arrivals, departures, and approaches. This work will impact the design and evaluation of both paper and electronic aeronautical charts. Our goal is to understand what information the pilot needs so that qualified pilots can reliably use charts with detailed PBN-related information. Well-managed and well-executed research in this area will support a successful transition to NextGen.

\section{References}

[1] Instrument Procedures Handbook, FAA, Washington, DC, FAA-H-8261-1A, 2007, Available: www.faa.gov.

[2] Instrument Flying Handbook, FAA, Washington, DC, FAA-H-8083-15A, 2008, Available: www.faa.gov.

[3] AVS Work Plan for NextGen 2012, FAA, Washington, DC, 2012, Available:

http://www.faa.gov/nextgen/media/avs nextgen wor kplan_2012.pdf

[4] NextGen Implementation Plan 2012, FAA, Washington, DC, 2012, Available:

www.faa.gov/nextgen/implementation/plan.

[5] Roadmap for Performance-based Navigation Version 2. FAA, Washington, DC, 2006, Available: www.faa.gov.

[6] Performance Based Navigation Capabilities Report. MITRE CAASD, McLean, VA, 2011, Available:

http://www.mitrecaasd.org/PBNCapabilityReport/

[7] NextGen Integration and Implementation Office Response to Recommendations of the RTCA NextGen Mid-Term Implementation Task Force, FAA, Washington, DC, 2010. 
[8] US Terminal and En Route Area Navigation Operations, Advisory Circular 90-100A, FAA, Washington, DC, 2007, Available: www.faa.gov.

[9] Approval Guidance for RNP Operations and Barometric Vertical Navigation in the U.S. National Airspace System. Advisory Circular 90-105, FAA, Washington, DC, 2009, Available: .

[10] Approval Guidance for RNP Procedures with Special Aircraft and Aircrew Authorization Required (AR), Advisory Circular 90-101A, FAA, Washington, DC, 2011, Available: www.faa.gov.

[11] R. Barhydt, and C. Adams, "Human Factors Considerations for Performance-Based Navigation." NASA, Langley, VA, NASA/TM-2006-214531, 2006. Available: http://ntrs.nasa.gov/

[12] R. Barhydt and C. Adams. "Human Factors Considerations for Area Navigation Departure and Arrival Procedures." Presented at 25th Congress of the International Council of the Aeronautical Sciences, Hamburg, Germany, 2006.

[13] A. Butchibabu, A. Midkiff, A. Kendra, R.J. Hansman, and D.C. Chandra, Analysis of Safety Reports Involving Area Navigation and Required Navigation Performance Procedures. Proceedings of the International Conference on Human-Computer Interaction in Aeronautics (HCI-Aero), 3-5 November 2010, Cape Canaveral, FL. Available: http://www.volpe.dot.gov.

[14] Electronic Display of Aeronautical Information (Charts), SAE International, Warrendale, PA, ARP5621, 2004.

[15] R. Ellerbrock, and S. Haffner, "Operational Efficiency of Dynamic Navigation Charting." Boeing Aero Quarterly Magazine, March 2012. Available: http://www.boeing.com/commercial/aeromagazine.

[16] D. Smith, "The ABCs of Jeppesen e-charts for the iPad." Avionics News. March, 2012.

[17] G. Larson, "Transformers: Jeppesen Unfolds a New Map." Business and Commercial Aviation, September, 2011.

Available: www.aviationweek.com/bca

[18] D. Chandra, R.J. Grayhem, and A. Butchibabu "Area Navigation and Required Navigation
Performance Procedures and Depictions," US DOT Volpe National Transportation Systems Center, Cambridge, MA, DOT/FAA/TC-12/8, 2012.

[19] A. Butchibabu, R.J. Grayhem, R. J., Hansman, and D. Chandra, Evaluating a De-cluttering Technique for NextGen RNAV and RNP Charts. Proceedings of $31^{\text {st }}$ Digital Avionics Systems Conference, 14-18 October 2012, Williamsburg, VA.

[20] A. Butchibabu, and R.J. Hansman, "Evaluating the Depiction of Complex RNAV/RNP Procedures and Analyzing a Potential De-Cluttering Technique," Massachusetts Inst. of Technology, Int. Center for Air Transportation, Technical Report ICAT 2012-3. May 10, 2012.

Available: http://hdl.handle.net/1721.1/70570

[21] D.C. Chandra, M. Yeh, V. Riley, and S.J. Mangold, "Human Factors Considerations in the Design and Evaluation of Electronic Flight Bags (EFBs), Version 2." USDOT Volpe Center: Cambridge, MA, DOT-VNTSC-FAA-03-07, 2003. Available: www.volpe.dot.gov

[22] Ricks, W.R., Jonsson, J.E., \& Barry, J.S. "Managing Approach Plate Information Study (MAPLIST): An Information Requirements Analysis of Approach Chart Use." NASA, Langley, VA, NASA Technical Paper 3561, 1996. Available: http://ntrs.nasa.gov/.

\section{Acknowledgements}

This paper was prepared by the Aviation Human Factors Division at the US DOT Volpe National Transportation Systems Center with funding from the FAA Human Factors Division (ANG-C1) in support of FAA Aviation Safety (AVS) and Flight Standards (AFS).

We thank Andrew Kendra and Alan Yost of the Volpe Center for their assistance on this project. We also thank Alan Midkiff, Abhizna Butchibabu, and John Hansman from the Massachusetts Institute of Technology (MIT) who contributed to this effort. MIT is funded by the Volpe Center (Contract DTR57-07-D-30006).

We thank the FAA program managers Dan Herschler and Tom McCloy and FAA technical sponsors Kathy Abbott and Mark Steinbicker for 
their assistance and guidance. John Moore, Brad Rush, and Valerie Watson from FAA AeroNav Products also contributed their valuable time and expertise to this effort. We also greatly value input from our mentors in industry, such as Ted Thompson (Jeppesen) and Pedro Rivas (Airline Pilots Association). Input from many private industry and government reviewers also helped to improve and strengthen our research plan.
The views expressed herein are those of the authors and do not necessarily reflect the views of the Volpe National Transportation Systems Center, the Research and Innovative Technology Administration, or the United States Department of Transportation.

31st Digital Avionics Systems Conference

October 14-18, 2012, Williamsburg, VA 